\title{
Predictors of warfarin non-adherence in younger adults after valve replacement surgery in the South Pacific
}

\author{
Linda J Thomson Mangnall, ${ }^{1,2}$ David W Sibbritt, ${ }^{3}$ Nihaya Al-Sheyab, ${ }^{2,4}$ \\ Robyn D Gallagher ${ }^{5}$
}

${ }^{1}$ Cardiothoracic Medical and Surgical Telemetry Unit, Sydney Adventist Hospital,

Wahroonga, New South Wales, Australia

${ }^{2}$ Faculty of Nursing and Midwifery, Charles Perkins Centre, University of Sydney, Sydney, New South Wales,

Australia

${ }^{3}$ Faculty of Health, University of Technology, Sydney, New South Wales, Australia

${ }^{4}$ Faculty of Nursing, Jordan University of Science and Technology, Irbid, Jordan

${ }^{5}$ Charles Perkins Centre and Sydney Nursing School,

University of Sydney,

Camperdown, New South

Wales, Australia

\section{Correspondence to} Linda J Thomson Mangnall, Charles Perkins Centre and Sydney Nursing School, University of Sydney,

Parramatta Road,

Camperdown, NSW 2050, Australia; linda.tm@sah.org.au

Received 21 March 2016 Revised 11 May 2016

Accepted 17 May 2016

\section{CrossMark}

To cite: Thomson Mangnall LJ, Sibbritt DW, Al-Sheyab $\mathrm{N}$, et al. Heart Asia 2016:8:18-23. doi:10.1136/heartasia-2016010751

\section{ABSTRACT}

Objectives Globally, mechanical valves are predominant as replacements for adolescents and younger adults with rheumatic heart disease (RHD). Mechanical valve implantation necessitates lifelong antithrombotic management (warfarin) and associated lifestyle modification, with event-free survival largely dependent on international normalised therapeutic ratios (INRs) remaining within the target therapeutic range. There is limited information on factors that may influence warfarin adherence among younger people or those in resource-limited settings. This study sought to identify predictors of warfarin adherence after valve replacement surgery for RHD in Fiji $(n=127)$.

Methods A cross-sectional study design was used. Results The sample had a mean age of 31.23 years (SD 13.34) and a mean time-since-surgery of 3.72 years (SD 3.95). Just over half were women $(n=71,56 \%)$ and almost two-thirds were indigenous (I-taukei, $n=78$, $61 \%)$. Most had an isolated valve procedure $(n=94$, $74 \%$ ) and at the time of survey, they were in New York Heart Association Class I ( $n=97,76 \%)$. A quarter $(n=33,26 \%)$ reported poor adherence with anticoagulation therapy and $13.38 \%(n=17)$ reported complete warfarin cessation. While younger age was significantly associated with non-adherence to warfarin therapy ( $p=0.008)$, the independent predictors of people who discontinue warfarin completely were those not understanding why warfarin was needed $(O R=9.97$, $p=0.006)$; a history of forgetting to take warfarin $(\mathrm{OR}=8.64, \mathrm{p}=0.0013)$ and travel time to heart clinic $>1$ hour $(\mathrm{OR}=5.80, \mathrm{p}=0.039)$.

Conclusions While medication adherence is complex and multifactorial, the consequences of warfarin nonadherence are potentially catastrophic. These results provide an important first step towards the development of country-specific and disease-specific strategies to improve warfarin adherence.

\section{INTRODUCTION}

Valvular heart disease remains a global health problem with increasingly different aetiologies between developed and low/middle-income countries. ${ }^{1-3}$ The most common aetiology in developed countries is age-related degenerative changes, whereas in low/middle-income countries, such as those in the South Pacific region, it is rheumatic heart disease (RHD), which primarily presents in adolescents and younger adults. ${ }^{1-3}$ RHD is particularly virulent in some South Pacific Island countries with surgical, or tertiary, intervention often required at much younger ages than other low/middle-income countries, and is therefore worthy of specific investigation. ${ }^{4}$ When surgical intervention is indicated, mechanical valve prostheses are more commonly implanted for reasons of durability and reduced lifetime reoperation requirements, but this also effectively exchanges one set of disease issues for those of another. ${ }^{15}$ Replacement of the malfunctioning valve/s normally improves quality of life and decreases medication requirements associated with a failing valve, but adds the lifelong requirement for antithrombotic management and the associated risk of severe adverse events such as stroke or haemorrhage. ${ }^{67}$ Currently, the only effective oral anticoagulation agent for mechanical valve prostheses are vitamin $\mathrm{K}$ antagonists in the form of warfarin. ${ }^{5} 7$ Consequently, antithrombotic health selfmanagement encompasses daily warfarin, regular medical visits to monitor international normalised therapeutic ratios (INRs), along with related dietary and lifestyle restrictions. ${ }^{7}$ Careful adherence to this regimen is critical to maintain the INR within the very narrow therapeutic window necessitated and there is a direct relationship between INR lability and decreased survival. ${ }^{7} 8$ This relationship was exemplified in a large prospective study of 1272 patients with isolated mechanical valves, the results of which demonstrated each $20 \%$ increase in INR lability equated with an $80 \%$ increase in mortality. ${ }^{8}$

Issues with adherence arise in all situations requiring the self-administration of treatment regardless of disease-type severity and health accessibility. ${ }^{9}{ }^{10}$ Data from the WHO suggest that adherence to long-term medication regimens averages $50 \%$ with even lower rates in low/middle-income countries. $^{9}$ Adherence is deemed the extent to which an individual's behaviour (eg, taking medication, following a diet, modifying habits, attending clinics) aligns with medical or health advice. ${ }^{9}$ 11 Conversely, non-adherence encompasses a diverse range of behaviours that lead to overuse or underuse of medications, non-attendance at clinics and ultimately threatens the health of an individual. ${ }^{9} 11$

There is a substantial body of research investigating factors that influence adherence; however, there is a great deal less research specific to warfarin adherence and this is more often situated in the pharmacological literature rendering it obscure to medical and nursing personnel. ${ }^{11-14}$ Predictors of warfarin adherence are inconsistent, being population dependent. For example, a low-income sample of Caucasian and African-American ethnicity at an oral anticoagulation clinic in the USA identified 
being divorced or never married and/or living in a shelter or with friends and relatives as predictors of non-adherence, but found no associations with education, gender, race or age. ${ }^{13}$ Conversely, a similar cohort (by age, race, gender, clinic) on the other side of the country but not in a low-income region found that an education level greater than high school and being currently employed were predictive of poorer adherence and also no associations with age, gender or race. ${ }^{14}$ While Waterman et $a l^{15}$ and Arnsten et $\mathrm{al}^{16}$ investigations both revealed that male gender and younger age were predictive of poorer adherence, with factors identified as significant in the aforementioned studies non-significant.

Intertwined with adherence issues is the fact that maintaining a therapeutic INR level is difficult and complex due to the multifactorial influences on efficacy and the burden of monitoring. ${ }^{10} 121517$ Consequently, even in well-resourced settings, only $65-75 \%$ of patients consistently maintain INRs within their targeted therapeutic range. ${ }^{15}{ }^{18}$ This rate reduces to around $50 \%$ in socioeconomically disadvantaged settings ${ }^{17}$ and decreases further to $12-17 \%$ in low/middle-income countries and indigenous communities. ${ }^{19}{ }^{20}$ At the patient level, factors that can influence the effectiveness of warfarin therapy include age (metabolic variations), diet, eating behaviour, weight gain/ weight loss, alcohol use, comorbidities and use of herbal products. ${ }^{10}$ Overlapping broader system issues also create barriers and this may relate to the drug (ie, cost, polypharmacy, availability); the patient (ie, deficits in support, knowledge and comprehension of repercussions, lifestyle restrictions); follow-up (ie, time constraints, delays in INR reporting and patient access) and health system (ie, warfarin management expertise, access, time consumed). ${ }^{10} 1521$ Low/middle-income countries with lessresourced healthcare systems have additional aspects to consider, including limited INR testing facilities and, often, an absence of anticoagulation specialist clinics. ${ }^{13}$

The Fiji Islands are a low/middle-income country in the South Pacific with a relatively large population of younger people who have had mechanical valves implanted for RHD. Thus, the purpose of this study was to explore antithrombotic-related health self-management after implantation of mechanical valve prosthesis for RHD and to determine if there are significant factors associated with warfarin adherence and/or self-cessation.

\section{Aims}

1. Describe self-reported adherence to, and knowledge of, antithrombotic therapy.

2. Identify significant factors associated with warfarin adherence and/or self-cessation

\section{METHODS}

The research reported here is part of an ongoing evaluation of healthcare outcomes for people undergoing valve surgery for RHD in Fiji. Detailed methodology has been described elsewhere ${ }^{6}$ but is summarised herein. The design of this component was cross-sectional.

\section{Participants}

All surviving and contactable Open Heart International (OHI) patients who had valve replacement (VR) surgery for RHD in Fiji from 1991 to 2014 inclusive, and aged 12 years or older at the time of survey, were contacted for inclusion in this study. National, and institutional, human research ethics committee approval was obtained in Australia and Fiji. All patients gave informed consent.

\section{Setting}

The Fiji Islands do not currently have an in-country cardiothoracic surgical service but accommodate fly-in/fly-out teams. One of these, an international volunteer, cardiothoracic surgical teams coordinated by $\mathrm{OHI}$ undertakes VR surgery at the Colonial War Memorial Hospital (CWMH), the national referral hospital located in Suva, Fiji. The majority of valve prostheses used are mechanical because they are cost-effective in the long term. Bioprostheses are offered to selected patients, such as young women who have not yet started, or completed their families, or people living in very remote areas. All patients implanted with mechanical prostheses require lifelong warfarin therapy for stroke prevention. ${ }^{25}$

Unlike more developed countries, access to warfarin and INR monitoring does not occur at a specialised warfarin outpatient clinic or by a visit to a nearby medical centre or medical practitioner. Instead, VR patients require a referral to cardiac, special out-patient clinics (SOPD) located at metropolitan and regional hospitals. Patients in more remote areas of the interior and/or outside of the two main islands of Viti Levu and Vanua Levu can have INRs collected at the provincial hospital facility but these are then sent to a tertiary centre for processing. Important aspects of the requirement to attend these SOPD relate to time, access and transport. Warfarin and other medications are free for all, if dispensed at the hospital on the clinic day. Supply issues can occur and patients must then buy or supplement available doses.

\section{Data collection}

Sociodemographic data, medical history, medications and disease-related information were obtained from the participant (including data from their personal INR and RHD clinic booklets) or hospital and clinic medical record/s (paper and electronic). Participants were interviewed at a location convenient for them, where upon they completed a health self-management questionnaire. Recruitment, data collection and survey administration occurred during seven field trips to Fiji over a 4-year period from April 2010 through April 2014.

\section{Self-reported, antithrombotic health self-management}

The antithrombotic health self-management questionnaire contained 14 questions. Ten questions related to oral anticoagulation in terms of knowledge, motivation, effects on daily life, adherence and blood testing and were adapted from van Doorn et $a l,^{22}$ with four additional questions related to heart clinic attendance. The complete 14-item questionnaire was piloted in Fiji during 2009 with minor changes made for the local vernacular. Patients, who had self-ceased their warfarin therapy, were asked to respond to questions from the perspective of when they were taking warfarin.

\section{Statistical analysis}

$\chi^{2}$ or Fisher's exact tests for categorical variables and independent samples t test for continuous variables were used to test for associations between demographic data, the health selfmanagement questionnaire responses and warfarin self-cessation. Logistic regression analysis was used to identify significant predictors of warfarin self-cessation. A $p$ value $\leq 0.05$ was considered significant. Multicollinearity was checked in the model diagnostic phase. None of the included variables were deemed to display significant multicollinearity. Statistical analyses were conducted using IBM SPSS Statistics (V.18) (PASW Statistics Chicago, Illinois, USA: SPSS Inc 2009). 


\section{RESULTS}

\section{Patient selection}

From 1991 to 2013, 204 patients had VR surgery for RHD by OHI of whom 127 surviving and contactable patients were enrolled in the study. There were no significant differences between those enrolled and those not, by gender $(p=0.053)$, ethnicity $(p=0.349)$, age $(p=0.612)$ and valve operated $(p=0.395)$. Participants were surveyed between 1 and 20 years postoperative with a mean of 3.72 years $(\mathrm{SD}=3.95)$ (table 1$)$ and a median of 2 years $(\mathrm{IQR}=4.0$ years).

\section{Patient characteristics}

Demographics

The mean age of the sample at the time of survey was 31.23 years $(\mathrm{SD}=13.34)$ (table 1$)$. Just over half of the sample were women $(n=71,56 \%)$ with a similar proportion being indigenous Fijian (I-taukei) ( $\mathrm{n}=78,62 \%)$. A quarter had completed high school $(n=30,24 \%)$ and over half were employed or still studying $(n=71,56 \%)$. Living locations were fairly evenly distributed between metropolitan, regional and rural/or other island (table 1) with three-quarters living within an hour's travel time of their SOPD $(n=99,78 \%)$.

\section{Clinical characteristics}

Most people had undergone an isolated procedure with $56 \%$ $(n=71)$ having mitral valve replacement and $18 \% \quad(n=23)$ having an aortic valve replacement (data not shown); the remainder $(n=33,26 \%)$ had two or more valves replaced (table 1). Three-quarters of participants were in New York Heart Association Functional Classification (NYHA) Class I at the time of survey $(n=97,76 \%)$. While all VR patients require RHD prophylaxis postoperatively through to 40 years of age,

Table 1 Sociodemographic, clinical characteristics and self-reported warfarin adherence of patients who had mechanical valve replacement for RHD in Fiji $(n=127)$

\begin{tabular}{|c|c|c|c|c|c|c|c|}
\hline & \multirow{2}{*}{\multicolumn{2}{|c|}{$\frac{\text { Sample }}{\text { Agree }}$}} & \multicolumn{5}{|c|}{ Self-ceased warfarin completely } \\
\hline & & & \multicolumn{2}{|c|}{ No $(n=110)$} & \multicolumn{2}{|c|}{ Yes $(n=17)$} & \multirow[b]{2}{*}{$p$ Value } \\
\hline & Mean & (SD) & Mean & (SD) & Mean & (SD) & \\
\hline \multicolumn{8}{|l|}{ Age } \\
\hline At the time of survey & 31.23 & $(13.34)$ & 32.45 & $(13.21)$ & 23.35 & $(11.68)$ & 0.008 \\
\hline \multicolumn{8}{|l|}{ Time } \\
\hline \multirow[t]{2}{*}{ Since surgery } & 3.72 & $(3.95)$ & 3.85 & $(4.16)$ & 2.85 & $(1.87)$ & 0.334 \\
\hline & $\mathbf{n}$ & $(\%)$ & $\mathbf{n}$ & $(\%)$ & $\mathbf{n}$ & $(\%)$ & p Value \\
\hline \multicolumn{8}{|l|}{ Gender } \\
\hline Female & 71 & (56) & 58 & (53) & 13 & (76) & 0.113 \\
\hline Male & 56 & (44) & 52 & (47) & 4 & (24) & \\
\hline \multicolumn{8}{|l|}{ Ethnicity } \\
\hline I-taukei & 78 & (61) & 64 & (58) & 14 & (82) & 0.065 \\
\hline Fijian of Indian descent or other & 49 & (39) & 46 & (42) & 3 & (18) & \\
\hline \multicolumn{8}{|l|}{ RHD prophylaxis } \\
\hline Yes & 38 & (30) & 32 & (29) & 6 & (35) & 0.603 \\
\hline No & 89 & (70) & 78 & (71) & 11 & (65) & \\
\hline \multicolumn{8}{|l|}{ Valve replaced } \\
\hline Isolated (mitral or aortic) & 94 & $(74)$ & 81 & (74) & 13 & (76) & 0.992 \\
\hline Multiple (>1 valve \pm tricuspid ring) & 33 & $(26)$ & 29 & (26) & 4 & (24) & \\
\hline \multicolumn{8}{|l|}{ NYHA at the time of survey } \\
\hline NYHA I & 97 & $(76)$ & 85 & (77) & 12 & (29) & 0.546 \\
\hline NYHA II or worse & 30 & (30) & 25 & (23) & 5 & (71) & \\
\hline \multicolumn{8}{|l|}{ Education } \\
\hline Did not/have not yet completed high school & 97 & $(76)$ & 84 & (76) & 13 & (76) & 0.992 \\
\hline Completed high school & 30 & (24) & 26 & (24) & 4 & (24) & \\
\hline \multicolumn{8}{|l|}{ Employment status } \\
\hline Not employed or studying & 56 & $(44)$ & 51 & (46) & 5 & (29) & 0.190 \\
\hline Employed or studying & 71 & (56) & 59 & (54) & 12 & (71) & \\
\hline \multicolumn{8}{|l|}{ Marital status } \\
\hline Single, separated, divorced, widowed & 67 & $(53)$ & 56 & (51) & 11 & (65) & 0.289 \\
\hline Married or cohabitating & 60 & (47) & 54 & (49) & 6 & (35) & \\
\hline \multicolumn{8}{|l|}{ Residence } \\
\hline Metropolitan & 44 & (34) & 40 & (36) & 4 & (24) & 0.523 \\
\hline Regional & 40 & $(31)$ & 33 & (30) & 7 & (41) & \\
\hline Rural/island & 45 & (35) & 37 & (34) & 6 & (35) & \\
\hline \multicolumn{8}{|l|}{ Time taken to get to clinic } \\
\hline 1 hour or less & 99 & $(78)$ & 89 & (81) & 10 & (59) & 0.058 \\
\hline More than 1 hour & 28 & (22) & 21 & (19) & 7 & (41) & \\
\hline
\end{tabular}


less than one-third $(\mathrm{n}=38,30 \%)$ had been continued, or recommenced, on this (table 1). While all patients had been commenced on oral warfarin anticoagulation therapy in hospital and discharged on same, almost one-in-eight $(\mathrm{n}=17,13.38 \%)$ reported self-cessation at the time of survey.

Patient and clinical characteristics and warfarin self-cessation Out of all the demographic and clinic characteristics (table 1), only younger age had a significant association with warfarin self-cessation $(p=0.008)$. Participants who had ceased taking warfarin altogether had a mean age of 23.35 years, almost 10 years younger, than those who remained on warfarin (mean 32.45 years).

Warfarin adherence, INR testing and clinic attendance

People who self-ceased warfarin were those who also reported multiple issues associated with aspects of warfarin ingestion (ie, taking, forgetting, bothered by, interfering with life, knowledge and fear, at $\mathrm{p}<0.03$ ) (table 2). There was a similar effect surrounding the mechanics and understanding of the regular INR testing (ie, pain, bothered by, interfering with life, knowledge) but only two of the aspects related to heart clinic attendance (ie, can't be bothered and knowledge) showed significant associations with warfarin self-cessation (table 2).

\section{Predictors of warfarin cessation}

A logistical regression analysis was undertaken to identify independent predictors of people who are more likely to discontinue warfarin. After multicollinearity eliminations and evaluation of theoretical and statistical significance, the variables of age-at-time-of-survey, gender and ethnicity, self-identified lack of understanding for the need to take warfarin, forgetting to take warfarin, employment status, time-since-surgery and travel time to heart clinic were entered as possible predictors table 3 ).

A lack of warfarin understanding $(\mathrm{p}=0.006)$, sometimes forgetting to take warfarin $(p=0.013)$ and a travel time to heart
Table 3 Independent predictors of warfarin self-cessation of patients who had mechanical valve replacement for RHD in Fiji $(n=127)$

\begin{tabular}{llll}
\hline Variable & OR & $95 \% \mathrm{Cl}$ & $\begin{array}{l}\mathbf{p} \\
\text { Value }\end{array}$ \\
\hline Age at the time of survey & 0.980 & $(0.907$ to 1.059) & 0.605 \\
Female gender & 2.915 & $(0.608$ to 13.983) & 0.181 \\
Fijian of Indian descent or other & 0.688 & $(0.106$ to 4.474) & 0.695 \\
Not understanding why warfarin needs & 9.975 & $(1.947$ to 51.099) & 0.006 \\
to be taken & & & \\
Sometimes forget to take warfarin & 8.641 & $(1.565$ to 47.725) & 0.013 \\
Employed or studying & 3.030 & $(0.053$ to 17.148) & 0.210 \\
Time since surgery & 0.873 & $(0.629$ to 1.211) & 0.417 \\
Time to travel to heart clinic $>1$ hour & 5.808 & $(1.094$ to 30.837) & 0.039 \\
\hline RHD, rheumatic heart disease. & & &
\end{tabular}

clinic of more than 1 hour were identified as being significantly associated with self-cessation of warfarin therapy. People who self-identify as not understanding why they need to take warfarin are nine times more likely to self-cease warfarin than those who do understand why they need to remain on it $(\mathrm{OR}=9.98$; CI 1.95 to 51.10); people who say they sometimes forget to take warfarin are eight times more likely to self-cease completely $(\mathrm{OR}=8.64$; CI 1.57 to 47.73) and those who have a travel time to clinic exceeding 1 hour are almost six times more likely to self-cease warfarin ( $\mathrm{OR}=5.81$; CI 1.09 to 30.84$)$ (table 3).

The Hosmer and Lemeshow test of the 'goodness-of-fit' suggests that this model is a good fit for the data $\left(\chi^{2} 3.545, p=0.896\right)$.

\section{DISCUSSION}

Adherence is a multidimensional issue and it has long been acknowledged there is not one optimal way to improve it. ${ }^{9}{ }^{11}$ Rather, a range of interventions need to be considered that factor in age and disease condition, the healthcare team,

Table 2 Antithrombotic health self-management and association with self-report of warfarin self-cessation of patients who had mechanical valve replacement for RHD in Fiji $(n=127)$

\begin{tabular}{|c|c|c|c|c|c|c|c|}
\hline & \multirow{2}{*}{\multicolumn{2}{|c|}{$\frac{\text { Sample }}{\text { Agree }}$}} & \multicolumn{4}{|c|}{ Self-ceased warfarin completely } & \multirow[b]{3}{*}{ p Value } \\
\hline & & & \multicolumn{2}{|c|}{ No $(n=110)$} & \multicolumn{2}{|c|}{ Yes $(n=17)$} & \\
\hline & $\mathbf{n}$ & $(\%)$ & $\mathrm{n}$ & $(\%)$ & $\mathrm{n}$ & $(\%)$ & \\
\hline \multicolumn{8}{|l|}{ Warfarin } \\
\hline Sometimes I can't be bothered taking & 33 & (26) & 20 & (18) & 13 & (76) & $<0.001$ \\
\hline Sometimes I forget to take & 36 & (28) & 22 & (20) & 14 & (82) & $<0.001$ \\
\hline Having to take daily warfarin tablets bothers me & 18 & (16) & 96 & (76) & 13 & (76) & $<0.001$ \\
\hline Having to remember to take daily warfarin tablets interferes with my life & 34 & (27) & 21 & (19) & 13 & (76) & $<0.001$ \\
\hline I don't understand the need for warfarin & 23 & (18) & 12 & (11) & 11 & (65) & $<0.001$ \\
\hline I am afraid I may bleed & 51 & (40) & 40 & (36) & 11 & (65) & 0.027 \\
\hline \multicolumn{8}{|l|}{ INR testing at heart clinic } \\
\hline The blood tests are painful & 72 & (57) & 58 & (53) & 14 & (82) & 0.022 \\
\hline Having to have regular blood tests bothers me & 38 & (34) & 77 & (61) & 12 & (71) & 0.005 \\
\hline Having to have blood tests interferes with my life & 68 & (54) & 54 & (49) & 14 & (82) & 0.010 \\
\hline I don't understand the need for these blood tests & 24 & (19) & 11 & (10) & 13 & (76) & $<0.001$ \\
\hline \multicolumn{8}{|l|}{ Heart clinic attendance } \\
\hline Sometimes I can't be bothered going & 32 & (26) & 24 & (22) & 8 & (47) & 0.026 \\
\hline Sometimes I forget to go & 14 & (11) & 10 & (9) & 4 & (24) & 0.094 \\
\hline Having to attend regular clinics interferes with my life & 88 & (69) & 75 & (68) & 13 & (76) & 0.491 \\
\hline I don't understand the need for heart clinic attendance & 14 & (11) & 9 & (8) & 5 & (29) & 0.022 \\
\hline
\end{tabular}


social, economic and country-specific conditions. ${ }^{9}{ }^{11}$ A key in understanding the determinants of poor adherence and/or selfcessation, and potentially finding ways to improve service delivery, is exploring the factors associated with it at the individual level. ${ }^{20}$ The finding of a significant association with younger age along with three independent predictors of warfarin selfcessation in this initial exploration of adolescents and younger adults after mechanical valve replacement surgery is an important first step towards this, as is identifying that almost one-in-eight people self-cease warfarin. This rate of warfarin self-cessation is remarkably low (17\%) although comparisons must be limited to because of the lack of data on similar populations. However, warfarin cessation rates are much higher at $22-33 \%$ in atrial fibrillation patients within the first year of commencing-despite AF also being a life-threatening illness. $^{12}{ }^{23}$ Nonetheless, the risk of catastrophic sequelae to warfarin non-adherence in the mechanical valve population is unacceptably high and any non-adherence is a cause for serious concern, particularly given the youthfulness of this sample. ${ }^{3} 7$

\section{Knowledge}

It is likely deficits in knowledge and understanding of warfarin therapy for all participants are underestimated, self-report notwithstanding. $^{24}$ The largest study until now by Chenot et al, ${ }^{24}$ evaluating safety-relevant knowledge of patients on warfarin found substantial gaps between how the patients rated themselves and actual measured knowledge. For example, while almost all patients (95\%) knew why they were on warfarin, 80\% did not know which non-prescription analgesic was safe to take with warfarin, and $68 \%$ did not know they had warfarin-related dietary restrictions or considerations. Importantly, many had a lack of awareness of the consequences of warfarin cessation and an understanding of the symptoms that indicated a likely stroke and thus the need for urgent medical attention, including paresis $(74 \%) .{ }^{24}$ Along with adherence to antithrombotic health selfmanagement, patient's knowledge and safety-relevant understanding of warfarin therapy is considered critical in preventing life-threatening complications. ${ }^{17}{ }^{25}$ This is borne out by data from a previous study of an intersecting sample of patients with mechanical VRs in Fiji that showed one-in-four had at least one morbidity event with oral anticoagulation-related events (bleeding or embolic) accounting for $58 \%$ of total morbidity events. ${ }^{6}$

\section{Forgetting to take}

While not quantified, one of the reasons patients gave for forgetting to take warfarin was the inconvenience of the dosing time. Fiji is a resource-limited country and like many other countries in similar circumstances, patients are more commonly managed by the more junior staff so both are given 'rules' for warfarin management without the adjunct of understanding the rationale. ${ }^{19}$ As an example, patients in Fiji are instructed to take their warfarin at 21:00 every night so for young people socialising in the evening, this time may not be appropriate.

In clinical practice, the potential effects of lifelong warfarin and self-management requirements, including risks of selfcessation, should be raised early with surgical candidates and their families along with collaborative advance planning to accommodate and/or modify current lifestyle incorporating key principles such as the 'right time to take warfarin, is the time you will always remember to take it'. ${ }^{20}$ Importantly, the language used in such discussions and subsequent interactions by all healthcare providers needs to reflect a shared responsibility for health, including avoidance of terminology such as 'compliant/non-compliant' which conveys judgement. ${ }^{20}$

\section{Travel time}

The identification of travel time greater than 1 hour as an independent predictor of warfarin self-cessation is unsurprising. In many Pacific Island counties, SOPD heart clinic attendance is intrinsically linked to warfarin access because it is at this clinic that INR testing is attended and where repeat warfarin prescriptions are issued with warfarin and other medications supplied free of charge, if dispensed at the hospital site. We used travel time instead of distance because 'time' is one of the recurring themes that patients consistently raise as a serious concern and the majority had no idea of the distance they travel; only the time it takes them. Nonetheless, using time as a proxy measure of distance still contrasted with that from a study of indigenous Australians investigating 'distance decay' as reason for poor attendance at INR clinics. ${ }^{26}$ In this context, 'distance decay' theorises that the travel-distance-to-clinic is inversely proportional to clinic attendance; thus, the greater the travel time, the lower the attendance rate. ${ }^{26}$ This was not found to be the case in the Australian study but was the case in ours. Possibly distance-to-clinic does not correlate to time-to-clinic because in the Australian indigenous sample the average distance to hospital-based clinic was $18 \mathrm{~km}$, which in that region would take half an hour, or less, even if by public transport, whereas $18 \mathrm{~km}$ in Fiji by public transport far exceeds an hour travel time (patient perspective and personal experience).

Additionally, in many low/middle-income countries, including Fiji, the time involved in clinic attendance can consume an entire day. While there is a lack of literature specific to VR patients, the burden of lengthy travel times to/from clinic, transport costs and other pressing issues such as a high unemployment rate, are similar to those experienced by many patients with chronic health self-management needs living in low/ middle-income countries, are recognised barriers to adherence and may be a further factor in self-cessation. ${ }^{26}$

\section{Study limitations and recommendations for further research}

The findings from this study may not be representative of all patients having VR for RHD in low/middle-income countries as only one surgical programme was included and follow-up was for a relatively short observational period of 4 years. There is a risk of potential bias with the use of opportunistic sampling, although this is largely mitigated because no potential participant declined involvement and the lack of differences between those enrolled and those not. While, statistically, there were adequate numbers of participants for the analysis conducted, the relatively small sample size restricted the number of predictor variables able to be included in the analysis. A number of measures, including warfarin adherence, were by self-report. Evaluating adherence and knowledge via self-report poses a risk of self-representational bias but also an increased likelihood that the reports of non-adherence and decreased knowledge are accurate. ${ }^{11}$ Most of these issues can be addressed with further research, which includes objective measurement of medication adherence and warfarin knowledge and a collaborative, multidisciplinary approach to identification and evaluation of other barriers to adherence. Furthermore, the relationship between INR variation and adverse outcomes should be addressed in a prospective study.

\section{CONCLUSION}

Our study found that almost one-in-eight participants selfceased their warfarin treatment with a significant association between younger age and warfarin self-cessation. Importantly, 
three independent predictors of warfarin self-cessation were identified, allowing appropriately targeted interventions to be now be developed and implemented.

\section{Key messages}

What is already known about this subject? Adherence to medication regimens, particularly over the longer-term and among younger people, is well recognised as problematic. While antithrombotic therapy encompasses adherence to warfarin medication, it also requires ongoing international normalised therapeutic ratio surveillance, dietary and lifestyle modifications to maintain target therapeutic ranges, thus adding further layers to adherence complexity. However, unlike many other adherence regimens, the personal consequences of non-adherence to antithrombotic therapy when living with a mechanical heart valve are eventually catastrophic.

\section{What does this study add?}

This study provides an initial baseline insight into levels of warfarin non-adherence and predictors that influence this among younger adults living with a mechanical valve in a less-resourced country. While it is well recognised that warfarin adherence is problematic even in well-resourced countries, there is scant work investigating this among younger adults, those with mechanical valves and those for whom access to the required medical surveillance may be limited.

How might this impact on clinical practice? Our outcomes suggest that additional attention be paid to patients' understandings of the need for warfarin therapy after mechanical valve placement along with the capacity to easily acquire follow-up prescriptions for warfarin after valve replacement.

Acknowledgements Each biannual field trip would not be possible without the efforts of a number of personnel. The authors wish to acknowledge: Michael Were, manager of Open Heart International $(\mathrm{OHI})$ and Melanie Windus, coordinator of $\mathrm{OHI}$, Fiji, for ongoing organisational and logistical support; Stephen Mangnall for ongoing, voluntary, technical and research-assistant support; the staff at the Colonial War Memorial, Lautoka and Labassa Hospital's (Fiji), Medical Record Departments, for their continued willingness to assist in paper and electronic patient record searches and the participants themselves, for so readily and openly, engaging with the researchers throughout the years of this long-term follow-up.

Contributors LITM contributed as primary investigator and writer to literature search, literature review, figures, study design, data collection, data entry, data analysis, data interpretation and HREC approval/s. DWS contributed to the statistical analyses and development of results and discussion. He revised the manuscript drafts. NAl-S contributed to the intellectual development, literature review, methods, discussion and revised manuscript drafts. RDG contributed to the intellectual development, literature review, methods, analysis and development of the results and discussion. She revised manuscript drafts.

Competing interests None declared.

Ethics approval The Adventist HealthCare Limited Ethics Committee (EC00141) and Fiji National Health Research Ethics Review Committee (FNHRERC).

Provenance and peer review Not commissioned; externally peer reviewed.

Data sharing statement All coauthors have access-by-request to the de-identified SPSS database for this research, which is stored in a password-protected computer of the primary author and in an encrypted-password-protected cloud storage (DropBox). Unpublished data include numerous incomplete data sets on variables such as medications, additional demographics (family living situation, education), open-ended questions responses (draft paper in progress using these data) and recently commenced collection of INR data.

\section{REFERENCES}

1 Carapetis J, Brown A, Walsh W, et al. Diagnosis and management of acute rheumatic fever and rheumatic heart disease in Australia. An evidence-based review. National Heart Foundation of Australia and the Cardiac Society of Australia and New Zealand, 2006.

2 World Health Organization. WHO expert consultation on rheumatic fever and rheumatic heart disease. WHO technical report series: 923. Geneva, Switzerland: World Health Organization, 2004.

3 Zühlke L, Engel ME, Karthikeyan G, et al. Characteristics, complications, and gaps in evidence-based interventions in rheumatic heart disease: the Global Rheumatic Heart Disease Registry (the REMEDY study). Eur Heart J 2015;36:1115-22.

4 Singh PIPK, Carapetis JR, Buadromo EM, et al. The high burden of rheumatic heart disease found on autopsy in Fiji. Cardiol Young 2008;18:62-9.

5 Nishimura RA, Otto CM, Bonow RO, et al. 2014 AHA/ACC guideline for the management of patients with valvular heart disease: executive summary: a report of the American College of Cardiology/American Heart Association Task Force on Practice Guidelines. J Am Coll Cardiol 2014;63:2438-88.

6 Thomson Mangnall L, Sibbritt D, Fry M, et al. Short- and long-term outcomes after valve replacement surgery for rheumatic heart disease in the South Pacific, conducted by a fly-in/fly-out humanitarian surgical team: a 20-year retrospective study for the years 1991 to 2011. J Thorac Cardiovasc Surg 2014;148:1996-2003.

7 lung $B$, Rodés-Cabau J. The optimal management of anti-thrombotic therapy after valve replacement: certainties and uncertainties. Eur Heart J 2014;35:2942-9.

8 Butchart EG, Payne N, Li H-H, et al. Better anticoagulation control improves survival after valve replacement. J Thorac Cardiovasc Surg 2002;123:715-23.

9 Sabate $\mathrm{E}(\mathrm{Ed})$. Adherence to long-term therapies: evidence for action. Geneva: World Health Organization; 2003. (Report available at: http://www.who.int/chp/ knowledge/publications/adherence_full_report.pdf).

10 Ansell J, Hirsh J, Poller L, et al. The pharmacology and management of the vitamin $\mathrm{K}$ antagonists: the Seventh ACCP Conference on Antithrombotic and Thrombolytic Therapy. Chest 2004;126:204S-33S

11 Horne R, Weinman J, Barber N, et al. Concordance, adherence and compliance in medicine taking. Report for the National Co-ordinating Centre for NHS Service Delivery and Organisation R \& D (NCCSDO). London, UK: NHS National Coordinating Centre for Service Delivery and Organisation (NCCSDO), 2005:1-311.

12 Kneeland PP, Fang MC. Current issues in patient adherence and persistence: focus on anticoagulants for the treatment and prevention of thromboembolism. Patient Prefer Adherence 2010;4:51-60.

13 Orensky IA, Holdford DA. Predictors of noncompliance with warfarin therapy in an outpatient anticoagulation clinic. Pharmacotherapy 2005;25:1801-8.

14 Platt $A B$, Localio $A R$, Brensinger $C M$, et al. Risk factors for nonadherence to warfarin: results from the IN-RANGE study. Pharmacoepidemiol Drug Saf 2008; 17:853-60.

15 Waterman AD, Milligan PE, Bayer L, et al. Effect of warfarin nonadherence on control of the International Normalized Ratio. Am J Health Syst Pharm 2004;61:1258-64.

16 Arnsten JH, Gelfand JM, Singer DE. Determinants of compliance with anticoagulation: a case-control study. Am J Med 1997;103:11-17.

17 Davis NJ, Billett HH, Cohen HW, et al. Impact of adherence, knowledge, and quality of life on anticoagulation control. Ann Pharmacother 2005;39:632-6.

18 Witt DM, Sadler MA, Shanahan RL, et al. Effect of a centralized clinical pharmacy anticoagulation service on the outcomes of anticoagulation therapy. Chest 2005;127:1515-22.

19 Ogendo SW. Pattern of anticoagulation control after heart valve surgery at the Kenyatta National Hospital, Nairobi. East Afr Med J 2000;77:354-8.

20 Wyber R, Grainger Gasser A, Thompson D, et al. Tools for Implementing RHD Control Programmes (TIPS) handbook. Perth, Australia: World Heart Federation and RhEACH, 2014

21 Ingelgard $\mathrm{A}$, Hollowell J, Reddy $\mathrm{P}$, et al. What are the barriers to warfarin use in atrial fibrillation?: Development of a questionnaire. J Thromb Thrombolysis 2006;21:257-65.

22 van Doorn C, Yates R, Tunstill A, et al. Quality of life in children following mitral valve replacement. Heart 2000;84:643-7.

23 Skeppholm M, Friberg L. Adherence to warfarin treatment among patients with atrial fibrillation. Clin Res Cardiol 2014;103:998-1005.

24 Chenot JF, Hua TD, Abu Abed M, et al. Safety relevant knowledge of orally anticoagulated patients without self-monitoring: a baseline survey in primary care. BMC Fam Pract 2014;15:104

25 Oterhals K, Deaton C, De Geest S, et al. European cardiac nurses' current practice and knowledge on anticoagulation therapy. Eur J Cardiovasc Nurs 2014;13:261-9.

26 Barth DD, Zühlke LJ, Joachim A, et al. Effect of distance to health facility on the maintenance of INR therapeutic ranges in rheumatic heart disease patients from Cape Town: no evidence for an association. BMC Health Serv Res 2015;15:219. 